\title{
Spin-Gravity Interactions and Equivalence Principle
}

\author{
Yu. N. Obukhov \\ Theoretical Physics Laboratory, Nuclear Safety Institute, Russian Academy of Sciences, \\ B. Tulskaya 52, 115191 Moscow, Russia \\ obukhov@ibrae.ac.ru \\ A. J. Silenko \\ Bogoliubov Laboratory for Theoretical Physics, Joint Institute for Nuclear Research, \\ 141980 Dubna, Russia \\ alsilenko@mail.ru \\ O. V. Teryaev \\ Bogoliubov Laboratory for Theoretical Physics, Joint Institute for Nuclear Research, \\ 141980 Dubna, Russia \\ teryaev@theor.jinr.ru \\ Published 29 February 2016
}

\begin{abstract}
The spin-gravity interactions imply the new manifestation of the equivalence principle leading to the absence of gravitoelectric and anomalous gravitomagnetic moments for fermions. This property is still valid in the presence of the space-time torsion due to the covariance arguments. The experimental bounds for the torsion, which may be extracted from modern co-magnetometer experiments, are discussed.
\end{abstract}

Keywords: Spin; Torsion; Magnetic and electric moments.

PACS numbers:04.20.Cv; 04.62.+v; 03.65.Sq

\section{Introduction}

The equivalence principle is one of the cornerstones of the relativistic gravity theory. ${ }^{1}$ One can recall the famous Einstein's discussion of a freely falling elevator in which gravitational effects are locally eliminated. The manifestations of the equivalence principle for macroscopical objects are well-tested ${ }^{1,2}$ and are even continued to the realm of elementary particles; ${ }^{3}$ for a recent review see. ${ }^{4}$

There is another manifestation of the equivalence principle related to the spin of a particle. It was probably for the first time discussed by Kobzarev and Okun ${ }^{5}$

This is an Open Access article published by World Scientific Publishing Company. It is distributed under the terms of the Creative Commons Attribution 3.0 (CC-BY) License. Further distribution of this work is permitted, provided the original work is properly cited. 
and also by Zakharov ${ }^{6}$ in ITEP (Moscow). In particular, the gravitoelectric and anomalous gravitomagnetic moments of a Dirac fermion was found to be zero. This property was later analyzed in connection with nucleon spin structure ${ }^{7}$ and was also generalized for the case of higher spins. ${ }^{7,8}$

The equivalence principle for the Dirac equation was also demonstrated in the pioneering work of Hehl and $\mathrm{Ni}^{9}$ where it is manifest in the appearance of a sum of the orbital and spin angular moments in the coupling to the gravity and inertia. It is very interesting to learn ${ }^{10}$ how the properties of the spin-gravity interactions are revealed in the framework of Poincaré gauge theory of gravity ${ }^{11}$ which is a natural extension of Einstein's general relativity (GR) theory based on the gauge-theoretic ideas. Poincaré gauge field potentials - the coframe and the Lorentz connection $\left(e_{i}^{\alpha}, \Gamma_{i}^{\alpha \beta}\right)$ - are coupled to the energy-momentum and spin tensors of matter. The corresponding Poincaré gauge field strengths are identified with the spacetime torsion and the curvature tensors $\left(T_{i j}{ }^{\alpha}, R_{i j}{ }^{\alpha \beta}\right)$, respectively. Our notations and conventions are given in. ${ }^{10}$

\section{Dirac Equation in Gauge Gravity Field}

Recasting the Dirac equation into the Schrödinder form $i \hbar \frac{\partial \psi}{\partial t}=\mathcal{H} \psi$, we find the Hermitian Hamiltonian ${ }^{12}$

$$
\begin{aligned}
\mathcal{H}= & \beta m c^{2} V+q \Phi+\frac{c}{2}\left(\pi_{b} \mathcal{F}^{b}{ }_{a} \alpha^{a}+\alpha^{a} \mathcal{F}^{b}{ }_{a} \pi_{b}\right) \\
& +\frac{c}{2}(\boldsymbol{K} \cdot \boldsymbol{\pi}+\boldsymbol{\pi} \cdot \boldsymbol{K})+\frac{\hbar c}{4}\left(\boldsymbol{\Xi} \cdot \boldsymbol{\Sigma}-\Upsilon_{\gamma_{5}}\right) .
\end{aligned}
$$

Here the kinetic 3-momentum operator $\pi_{a}=-i \hbar \partial_{a}-q A_{a}=p_{a}-q A_{a}$ accounts of the interaction with the electromagnetic field $A_{i}=\left(-\Phi, A_{a}\right)$, and we denoted

$$
\mathcal{F}^{b}{ }_{a}=V W^{b} \widehat{a}, \quad \Upsilon=V \epsilon^{\widehat{a} \widehat{a} \widehat{c}} \Gamma_{\widehat{a} \widehat{b} \widehat{c}}, \quad \Xi^{a}=\frac{V}{c} \epsilon^{\widehat{a} \widehat{a b} \widehat{c}}\left(\Gamma_{\widehat{0} \widehat{b} \widehat{c}}+\Gamma_{\widehat{b} \widehat{c} \widehat{0}}+\Gamma_{\widehat{c} \widehat{0} \widehat{b}}\right) .
$$

Equations (1) and (2) encompass the minimal coupling of both gravitational charges -translational (the mass or the energy-momentum) and rotational (the spin) onesto the Poincaré gauge field $\left(e_{i}^{\alpha}, \Gamma_{i}{ }^{\alpha \beta}\right)$; the functions $\left(V, W^{\widehat{a}}{ }_{b}, K^{a}\right)$ parametrize the components of the tetrad field via $V=e_{0}^{\widehat{0}}, W^{\widehat{a}}{ }_{b}=e_{b}^{\widehat{a}}, W^{\widehat{a}}{ }_{b} K^{b}=e_{0}^{\widehat{a}}$ and $e_{a}^{\widehat{0}}=0$.

As usual, $\alpha^{a}=\beta \gamma^{a}(a, b, c, \cdots=1,2,3)$ are the Dirac matrices and the spin matrices are defined by $\Sigma^{1}=i \gamma^{\hat{2}} \gamma^{\hat{3}}, \Sigma^{2}=i \gamma^{\hat{3}} \gamma^{\hat{1}}, \Sigma^{3}=i \gamma^{\hat{1}} \gamma^{\hat{2}}$ and $\gamma_{5}=i \alpha^{\hat{1}} \alpha^{\hat{2}} \alpha^{\hat{3}}$. Boldface notation is used for 3 -vectors $\boldsymbol{K}=\left\{K^{a}\right\}, \boldsymbol{\pi}=\left\{\pi_{a}\right\}, \boldsymbol{\alpha}=\left\{\alpha^{a}\right\}, \boldsymbol{\Sigma}=\left\{\Sigma^{a}\right\}$.

In order to make the coupling of spin and torsion explicit, we now use the decomposition of the connection into the Riemannian and post-Riemannian parts ${ }^{10}$

$$
\Upsilon=\widetilde{\Upsilon}+V c \check{T}^{\widehat{0}}, \quad \Xi^{\widehat{a}}=\widetilde{\Xi}^{\widehat{a}}-V \check{T}^{\widehat{a}} .
$$

The tilde denotes the Riemannian quantities

$$
\widetilde{\Upsilon}=V \epsilon^{\widehat{a} \widehat{b} \widehat{c}} \widetilde{\Gamma}_{\widehat{a} \widehat{b} \widehat{c}}=-V \epsilon^{\widehat{a} \widehat{b} \widehat{c}} \mathcal{C}_{\widehat{a} \widehat{b} \widehat{c}}, \quad \widetilde{\Xi}_{\widehat{a}}=\frac{V}{c} \epsilon_{\widehat{a} \widehat{b} \widehat{c}} \widetilde{\Gamma}_{\widehat{0}}{ }_{\widehat{b} \widehat{c}}=\epsilon_{\widehat{a} \widehat{b} \widehat{c}} \mathcal{Q}^{\widehat{b} \widehat{c}},
$$

which are constructed in terms of the following auxiliary objects: 


$$
\begin{aligned}
& \mathcal{Q}_{\widehat{a} \widehat{b}}=g_{\widehat{a} \widehat{c}} W_{\widehat{b}}^{d_{\widehat{b}}}\left(\frac{1}{c} \dot{W}^{\widehat{c}}{ }_{d}+K^{e} \partial_{e} W^{\widehat{c}}{ }_{d}+W^{\widehat{c}}{ }_{e} \partial_{d} K^{e}\right), \\
& \mathcal{C}_{\widehat{a} \widehat{b}}^{\widehat{c}}=W^{d}{ }_{\widehat{a}} W_{\widehat{b}}^{e_{\widehat{b}}} \partial_{[d} W^{\widehat{c}}{ }_{e]}, \quad \mathcal{C}_{\widehat{a} \widehat{b} \widehat{c}}=g_{\widehat{c} \widehat{d}} \mathcal{C}_{\widehat{a} \vec{b}} \widehat{d}^{\widehat{d}} .
\end{aligned}
$$

The dot ' denotes the derivative with respect to the coordinate time $t$. Naturally, we interpret $\mathcal{C}_{\widehat{a} \widehat{b}}{ }^{\widehat{c}}=-\mathcal{C}_{\widehat{b} \widehat{a}}{ }^{\widehat{c}}$ as the anholonomity object for the spatial triad $W^{\widehat{a}}{ }_{b}$. The non-Riemannian parts in (3) are constructed from the axial torsion vector

$$
\check{T}^{\alpha}=-\frac{1}{2} \eta^{\alpha \mu \nu \lambda} T_{\mu \nu \lambda}
$$

where $\eta^{\alpha \mu \nu \lambda}$ is the totally antisymmetric Levi-Civita tensor.

As a result, we can explicitly identify the spin-torsion coupling

$$
-\frac{\hbar c V}{4}\left(\boldsymbol{\Sigma} \cdot \check{\boldsymbol{T}}+c \gamma_{5} \check{T}^{\hat{0}}\right)
$$

which comes from the last terms of the Dirac Hamiltonian (1). As usual, $\check{\boldsymbol{T}}=\left\{\check{T}^{a}\right\}$.

The semiclassical limit is derived with the help of the Foldy-Wouthuysen (FW) transformation leading to FW Hamiltonian:

$$
\mathcal{H}_{F W}=\mathcal{H}_{F W}^{(1)}+\mathcal{H}_{F W}^{(2)}+\mathcal{H}_{F W}^{(3)} .
$$

The three terms read, respectively,

$$
\begin{aligned}
\mathcal{H}_{F W}^{(1)}= & \beta \epsilon^{\prime}+\frac{\hbar c^{2}}{16}\left\{\frac{1}{\epsilon^{\prime}},\left(2 \epsilon^{c a e} \Pi_{e}\left\{p_{b}, \mathcal{F}^{d}{ }_{c} \partial_{d} \mathcal{F}^{b}{ }_{a}\right\}+\Pi^{a}\left\{p_{b}, \mathcal{F}^{b}{ }_{a} \widetilde{\Upsilon}\right\}\right)\right\} \\
& +\frac{\hbar m c^{4}}{4} \epsilon^{c a e} \Pi_{e}\left\{\frac{1}{\mathcal{T}},\left\{p_{d}, \mathcal{F}^{d}{ }_{c} \mathcal{F}^{b}{ }_{a} \partial_{b} V\right\}\right\} \\
\mathcal{H}_{F W}^{(2)}= & \frac{c}{2}\left(K^{a} p_{a}+p_{a} K^{a}\right)+\frac{\hbar c}{4} \Sigma_{a} \widetilde{\Xi}^{a} \\
& +\frac{\hbar c^{2}}{16}\left\{\frac{1}{\mathcal{T}},\left\{\Sigma_{a}\left\{p_{e}, \mathcal{F}^{e}{ }_{b}\right\},\left\{p_{f},\left[\epsilon^{a b c}\left(\frac{1}{c} \dot{\mathcal{F}}^{f}{ }_{c}-\mathcal{F}^{d}{ }_{c} \partial_{d} K^{f}+K^{d} \partial_{d} \mathcal{F}^{f}{ }_{c}\right)\right.\right.\right.\right. \\
& \left.\left.\left.\left.-\frac{1}{2} \mathcal{F}^{f}{ }_{d}\left(\delta^{d b} \widetilde{\Xi}^{a}-\delta^{d a} \widetilde{\Xi}^{b}\right)\right]\right\}\right\}\right\}, \\
\mathcal{H}_{F W}^{(3)}= & \frac{\hbar}{2} \Sigma^{a} \Omega_{a}^{(T)} .
\end{aligned}
$$

The curly brackets $\{$,$\} denote anticommutators and we introduced the operators$

$$
\begin{aligned}
\Omega_{a}^{(T)}= & -\frac{c}{2} V \delta_{a b} \check{T}^{\hat{b}}+\beta \frac{c^{3}}{8}\left\{\frac{1}{\epsilon^{\prime}},\left\{p_{b}, \mathcal{F}^{b}{ }_{a} V \check{T}^{\hat{0}}\right\}\right\} \\
& +\frac{c^{2}}{16}\left\{\frac{1}{\mathcal{T}},\left\{\left\{p_{e}, \mathcal{F}^{e}{ }_{b}\right\},\left\{p_{f}, \mathcal{F}^{f}{ }_{d} V\left(\delta^{d b} \check{T}^{\hat{a}}-\delta^{d a} \check{T}^{\hat{b}}\right)\right\}\right\}\right\},
\end{aligned}
$$




$$
\epsilon^{\prime}=\sqrt{m^{2} c^{4} V^{2}+\frac{c^{2}}{4} \delta^{a c}\left\{p_{b}, \mathcal{F}_{a}^{b}\right\}\left\{p_{d}, \mathcal{F}_{c}{ }_{c}\right\}}, \quad \mathcal{T}=2 \epsilon^{\prime 2}+\left\{\epsilon^{\prime}, m c^{2} V\right\} .
$$

The first two terms (10) and (11) determine the dynamics of the Dirac fermion on the Riemannian spacetime manifold, whereas (12) with (13) gives the general description of the contribution of torsion field to the FW Hamiltonian.

The equation of spin motion is obtained from the commutator of the FW Hamiltonian with the polarization operator $\boldsymbol{\Pi}=\beta \boldsymbol{\Sigma}$ :

$$
\frac{d \boldsymbol{\Pi}}{d t}=\frac{i}{\hbar}\left[\mathcal{H}_{F W}, \boldsymbol{\Pi}\right]=\mathbf{\Omega} \times \mathbf{\Pi} .
$$

As a special case, let us consider the flat Minkowski metric with $V=1, K^{a}=$ $0, W^{\widehat{a}}{ }_{b}=\delta_{b}^{a}$. The spin precesses under the action of the torsion with the angular velocity $\boldsymbol{\Omega}=\boldsymbol{\Omega}^{(T)}$, where

$$
\boldsymbol{\Omega}^{(T)}=-\frac{c}{2} \check{\boldsymbol{T}}+\beta \frac{c^{3}}{8}\left\{\frac{1}{\epsilon^{\prime}},\left\{\boldsymbol{p}, \check{T}^{\hat{0}}\right\}\right\}+\frac{c}{8}\left\{\frac{c^{2}}{\epsilon^{\prime}\left(\epsilon^{\prime}+m c^{2}\right)},\left(\left\{\boldsymbol{p}^{2}, \check{\boldsymbol{T}}\right\}-\{\boldsymbol{p},(\boldsymbol{p} \cdot \check{\boldsymbol{T}})\}\right)\right\} .
$$

\section{Gravitomagnetic and Gravitoelectric Moments}

The covariance arguments impose strong constraints on the possible structure of the nonminimal coupling, and in particular on the gravitoelectric and gravitomagnetic moments. The electromagnetic field strength $F_{i j}$ is tensor and hence the Pauli term $\sigma^{\alpha \beta} F_{\alpha \beta}=\sigma^{i j} F_{i j}$ is invariant under arbitrary coordinate and Lorentz frame transformations. In contrast, the local Lorentz connection $\Gamma_{i \alpha \beta}$ is not a tensor, and the same applies to $\Phi_{\alpha \beta}=\left\{\pi^{i}, \Gamma_{i \alpha \beta}\right\} /(2 m)$ which can be interpreted as a (weak) gravitomagnetic field. Hence the Pauli-type term $\sigma^{\alpha \beta} \Phi_{\alpha \beta}$ is not an invariant object. An attempt to extend $\sigma^{\alpha \beta} \Phi_{\alpha \beta}$ via the identity ${ }^{12}$

$$
g^{i j}\left(\hbar^{2} D_{i} D_{j}+\pi_{i} \pi_{j}\right)=\frac{\hbar m}{2}\left[\sigma^{\alpha \beta} \Phi_{\alpha \beta}-\frac{\hbar}{8 m}\left(2 \Gamma^{i}{ }_{\alpha \beta} \Gamma_{i}^{\alpha \beta}+i \varepsilon^{\alpha \beta \mu \nu} \Gamma_{\alpha \beta}^{i} \Gamma_{i \mu \nu} \gamma_{5}\right)\right]
$$

also fails since both sides are non-covariant in view of the non-covariance of $\pi_{i}$.

We thus conclude that the anomalous gravitomagnetic moment is not allowed in the covariant Dirac-Pauli theory with a nonminimal coupling of a fermion to the Poincaré gauge gravitational field. This demonstrates a limited nature of analogies between gravitational and electromagnetic interactions observed in the weakfield approximation. The same conclusion is valid for the anomalous gravitoelectric moment. It is worthwhile to recall that the weak-field analysis of the gravitational form-factors of Dirac fermions by Kobzarev and Okun ${ }^{5}$ have shown that the anomalous gravitomagnetic and gravitoelectric moments should be strictly zero due to the equivalence principle. Thus we now have its natural generalization to the case of strong gauge gravitational fields. 


\section{Experimental Bounds on Spin-Torsion Coupling}

To determine bounds on the spacetime torsion from the experiments with comagnetometers, ${ }^{13}$ we present ${ }^{10}$ the classical limit of FW Hamiltonian as follows

$$
H=-g_{N} \frac{\mu_{N}}{\hbar} \boldsymbol{B} \cdot s-\boldsymbol{\omega} \cdot s-\frac{c}{2} \check{\boldsymbol{T}} \cdot \boldsymbol{s},
$$

where $g_{N}$ is the nuclear $g$-factor and $\mu_{N}$ is the nuclear magneton.

Let us denote two kinds of atoms $\left({ }^{199} \mathrm{Hg}\right.$ and $\left.{ }^{201} \mathrm{Hg}\right)$ by the subscripts 1 and 2. The measured ratio of Zeeman frequencies for transitions between neighbouring atomic levels, $R=\nu_{2} / \nu_{1}$, depends on the direction of the magnetic field $\boldsymbol{B}$ and on the spin-torsion coupling. Two opposite directions of the magnetic field were used in the actual experiment. ${ }^{13}$ The calculation ${ }^{14}$ of the difference of these ratios for the two opposite directions of magnetic field yields

$$
R_{+}-R_{-}= \pm \frac{1-\mathcal{G}}{\nu_{1}}\left[2 f \cos \theta+\frac{c}{2 \pi}|\check{T}| \cos \Theta\right], \quad \mathcal{G}=\frac{g_{2}}{g_{1}} .
$$

Here $\theta$ is the angle between the direction of the magnetic field and the Earth's rotation axis, whereas $\Theta$ is the angle between $\boldsymbol{B}$ and the torsion $\check{\boldsymbol{T}}, f=\omega /(2 \pi)$ is the Earth's rotation frequency, and $\nu_{1}$ is the Zeeman frequency for atoms of the first kind. The experimental conditions ${ }^{13}$ for ${ }^{199} \mathrm{Hg}$ and ${ }^{201} \mathrm{Hg}$ atoms correspond to the angle $\theta \approx 0$, and the ratio of $g$-factors $\mathcal{G}=-0.369139$. Using the experimental data, ${ }^{13}$ we then obtain the restriction on the absolute value of the spacetime torsion:

$$
\frac{\hbar c}{4}|\check{\boldsymbol{T}}| \cdot|\cos \Theta|<2.2 \times 10^{-21} \mathrm{eV}, \quad|\check{\boldsymbol{T}}| \cdot|\cos \Theta|<4.3 \times 10^{-14} \mathrm{~m}^{-1} .
$$

We can perform a similar analysis of the other experiments ${ }^{15-17}$ where the difference of the weighted Zeeman frequencies were measured for He and Xe atoms:

$$
|\Delta \omega|=\left|\omega_{2}-\mathcal{G} \omega_{1}\right|=\left|(1-\mathcal{G})\left(\omega \cos \theta+\frac{c}{2}|\check{T}| \cos \Theta\right)\right|, \quad \mathcal{G}=\frac{g_{2}}{g_{1}} .
$$

Specifically, by making use of the experimental data, ${ }^{15}$ we extract the new restriction on the minimal coupling of torsion (with the $g$-factor ratio $\mathcal{G}=g_{\mathrm{He}} / g_{\mathrm{Xe}}=$ $2.75408159(20)$, and $\left.(1-\mathcal{G}) \omega \cos \theta=-6.87263 \times 10^{-5} \mathrm{rad} / \mathrm{s}\right)$ :

$$
\frac{\hbar c}{2}|\check{\boldsymbol{T}}| \cdot|(1-\mathcal{G}) \cos \Theta|<4.1 \times 10^{-22} \mathrm{eV}, \quad|\check{\boldsymbol{T}}| \cdot|\cos \Theta|<2.4 \times 10^{-15} \mathrm{~m}^{-1} .(22
$$

Equations (20) and (22) present the new strong bounds on the spacetime torsion.

\section{Conclusions and Discussion}

The equivalence principle is manifest in spin-gravity interactions as the absence of the gravitoelectric and anomalous gravitomagnetic moments of fermions. This result is valid in Einstein's general relativity and it is naturally generalized to the Poincaré gauge gravitational theory.

We have shown that the strong bounds for the spacetime torsion can be obtained via the re-analysis of the data of modern co-magnetometer experiments. Moreover, 
the new results presented at this conference ${ }^{18}$ may be probably used to improve even further these bounds by about an order of magnitude, although some care is definitely required. ${ }^{19,20}$

\section{Acknowledgments}

We are grateful to F.W. Hehl and S. Karpuk for the valuable discussions and correspondence. O.T. is indebted to the organizers, especially to Haiyan Gao and BoQiang Ma, for the warm hospitality in Beijing, and to the participants, especially to Wei-Tou Ni, M. Snow and F. Allmendinger for the helpful discussions.

The work was supported in part by RFBR Grant 14-01-00647.

\section{References}

1. C.M. Will, Living Rev. Relativity 9, 3 (2006); http://www.livingreviews.org/lrr-20063

2. T. Damour, Class. Quantum Grav. 29, 184001 (2012).

3. V. V. Nesvizhevsky, Mod. Phys. Lett. A 27, 1230006 (2012).

4. W.-T. Ni, Rep. Prog. Phys. 73, 056901 (2010).

5. I. Y. Kobzarev and L. B. Okun, Zh. Eksp. Teor. Fiz. 43, 1904 (1962) [Sov. Phys. JETP 16, 1343 (1963)].

6. I. Y. Kobsarev and V. I. Zakharov, Phys. Lett. B 30, 488 (1969); Ann. Phys. (USA) 60, 448 (1970).

7. O. V. Teryaev, Spin structure of nucleon and equivalence principle, hep-ph/9904376.

8. O. V. Teryaev, AIP Conf. Proc. 915, 260 (2007) [hep-ph/0612205]; Mod. Phys. Lett. A 24, 2831 (2009).

9. F. W. Hehl and W.-T. Ni, Phys. Rev. D 42, 2045 (1990).

10. Y. N. Obukhov, A. J. Silenko and O. V. Teryaev, Phys. Rev. D 90, 124068 (2014).

11. M. Blagojević and F.W. Hehl (eds.), Gauge Theories of Gravitation. A Reader with Commentaries (Imperial College Press, London, 2013)

12. Yu.N. Obukhov, A.J. Silenko, and O.V. Teryaev, Phys. Rev. D 84, 024025 (2011).

13. B. J. Venema, P. K. Majumder, S. K. Lamoreaux, B. R. Heckel, and E. N. Fortson, Phys. Rev. Lett. 68, 135 (1992).

14. A.J. Silenko and O.V. Teryaev, Phys. Rev. D 76, 061101(R) (2007).

15. C. Gemmel et al., Eur. Phys. J. D 57, 303 (2010).

16. C. Gemmel et al., Phys. Rev. D 82, 111901(R) (2010).

17. M. Burghoff et al., J. Phys.: Conf. Ser. 295, 012017 (2011).

18. F. Allmendinger, W. Heil, S. Karpuk, W. Kilian, A. Scharth, U. Schmidt, A. Schnabel and Y. Sobolev et al., Phys. Rev. Lett. 112, 110801 (2014); F. Allmendinger, These Proceedings.

19. M. V. Romalis, D. Sheng, B. Saam and T. G. Walker, Phys. Rev. Lett. 113, 188901 (2014).

20. F. Allmendinger, U. Schmidt, W. Heil, S. Karpuk, A. Scharth, Y. Sobolev and K. Tullney, Phys. Rev. Lett. 113, 188902 (2014). 